EPJ Web of Conferences 59, 14007 (2013)

DOI: $10.1051 /$ epjconf/20135914007

(C) Owned by the authors, published by EDP Sciences, 2013

\title{
Equation of state and opacities for warm dense matter
}

\author{
Manuel Cotelo ${ }^{1, a}$, Alberto G. de la Varga ${ }^{1}$, Pedro Velarde ${ }^{1,2}$ \\ and François de Gaufridy ${ }^{1,3}$ \\ ${ }^{1}$ Instituto de Fusión Nuclear, UPM, Madrid, Spain \\ ${ }^{2}$ Laboratoire d'Optique Applique, UMR CNRSIENSTA ParisTech/Ecole Polytechnique \\ ParisTech, Chemin de la hunière, 91671 Palaiseau, France \\ ${ }^{3}$ Institute of Physics, Czech Academy of Sciences, Prague, Czech Republic
}

\begin{abstract}
This work presents recent developments in the calculation of opacity and equation of state tables suitable for including in the radiation hydrodynamic code ARWEN [1] to study processes like ICF or X-ray secondary sources. For these calculations we use the code bigbart to compute opacities in LTE conditions, with self-consistent data generated with the Flexible Atomic Code (FAC) [2]. Non-LTE effects are approximately taken into account by means of the new RADIOM model developed in [3], which makes use of existing LTE data tables. We use the screened-hydrogenic model [4] to derive the Equation of State (EOS) using the population and energy of each level.
\end{abstract}

\section{INTRODUCTION}

A complete study of an ICF target includes a great variety of different physical processes that involve matter at extreme conditions, moving from room temperature to several hundreds of electronvolts and reaching densities up to a hundred times the solid density. Accurate knowledge of the relevant properties of the materials involved in the ICF process in such a wide range of temperature-density states is a key issue to get realistic computer simulations and then improve our understanding of the process.

This work describes a methodology to obtaind EOS and opacity tables suited for radiation hydrodynamic codes like ARWEN [1] with consistent data between thermodynamics and atomic calculations. The challenge is to find the correct balance between accuracy and computational cost. We will show recent improvements in the bigbart opacity code [5] and study non-local thermodynamic equilibrium (NLTE) effects using the RADIOM model and also derive an EOS from atomic calculations.

\section{RADIATIVE MODEL}

Calculating the radiative properties of a plasma is a formidable task, even further if NLTE effects are included in our models, where a myriad of transitions between levels are needed for solving a collisional-radiative system with the increasing complexity of ions with increasing Z. The simplest codes assume an average atom and calculates transitions between average orbitals. Other models merge close configurations to obtain configuration-averages reducing the amount of levels to treat. Both possibilities are implemented in our code bigbart to compute the spectral opacity and emissivity of a plasma for a given temperature and density.

\footnotetext{
${ }^{\mathrm{a}} \mathrm{e}$-mail: manuel. cotelo@upm.es
}

This is an Open Access article distributed under the terms of the Creative Commons Attribution License 2.0, which permits unrestricted use, distribution, and reproduction in any medium, provided the original work is properly cited. 


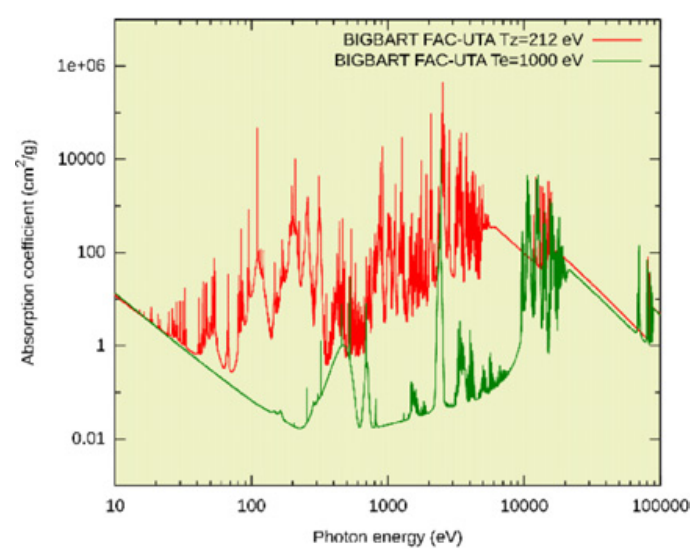

(a) Absorption, in $\mathrm{cm}^{2} / g$.

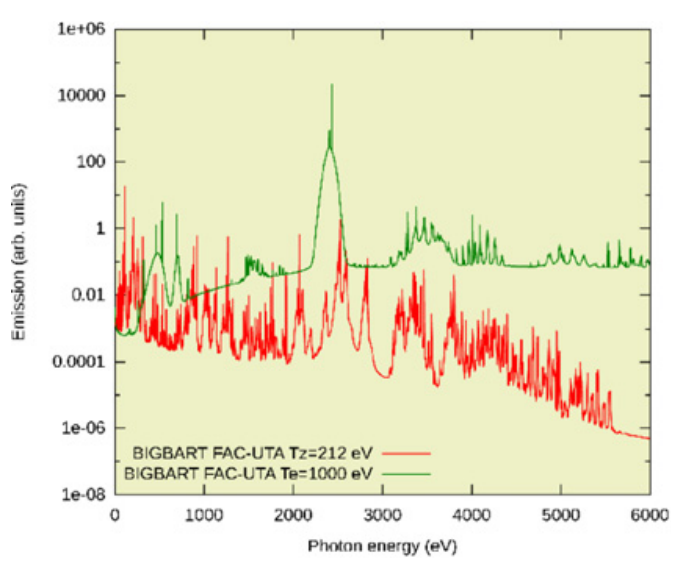

(b) Emission, in arbitrary units

Figure 1. Radiative properties for $\mathrm{Au}$ at $19 \mu \mathrm{g} / \mathrm{cm}^{3}$ and temperature of $1000 \mathrm{eV}$ (LTE data in green, NLTE data in red). The actual temperature of the Au plasma in NLTE conditions is $T_{e}=1000 \mathrm{eV}$ while the RADIOM model gives an LTE equivalen temperature of $T_{z}=212 \mathrm{eV}$ used to compute NLTE radiation coefficients from previous existing LTE data.

In order to reduce computational cost as much as possible bigbart now uses modified FAC output files to reuse the atomic data generated. Energy levels, radiative transitions and photoionization crosssections are calculated under request and stored in indexed binary files. This way, when generating opacity tables, the data generated in a temperature-density point is used, accessing and extracting necessary self-consistent data.

Energy levels are applied in a full Saha system that yields the fractional ionization occupancies. Ionization potentials are taken from literature $[6,7]$ when possible or calculated with FAC. Ionization potential lowering is accounted for using the modified Stewart-Pyatt formula [8]. Mixtures are treated consistently, iterating until both chemical potential and intrinsic densities converge.

A Voigt profile is used for line broadening with Doppler [9] and UTA Gaussian widths, and natural [9] and collisional [10] Lorentzian widths.

\subsection{NLTE effects}

The RADIOM model mimicks NLTE properties at temperature $T_{e}=T_{e}\left(Z^{*}, E O S\right.$, opacity) (where $Z^{*}$ is the average charge state) with LTE calculations at an equivalent temperature $T_{z}$. This is achieved by fitting the maximum of the charge state distribution in NLTE at $T_{e}$ in an extended Saha equation with the LTE value at $T_{z}$ with the standard Saha equation.

In figure 1 is shown the absorption coefficient and the emission of gold at $1000 \mathrm{eV}$ for LTE and NLTE using the RADIOM model, both pictures use the UTA model whithin FAC atomic package. The absorption coefficient for NLTE is higher than in LTE and the emission in NLTE is lower, as expected because in NLTE the excited states of the atom are less populated than in LTE and then emission is lowered.

\section{EQUATION OF STATE}

The screened-hydrogenic model (SHM) described in [4] is used to obtain the EOS for LTE conditions. The Helmholtz free energy $F=F(\rho, T)$ is computed from the electronic configuration of the average atom and then it is possible to derive a thermodynamic consistent EOS applying the Maxwell's relations. 


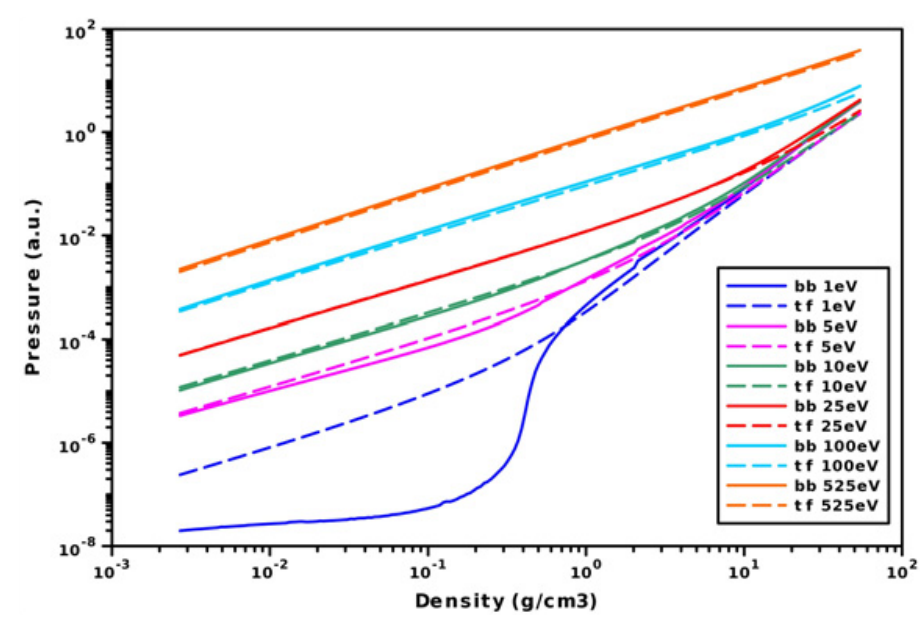

Figure 2. Pressure of Al using two different models to compute the chemical potential, Thomas-Fermi (tf in the figure) model and SHM (bb in the figure).

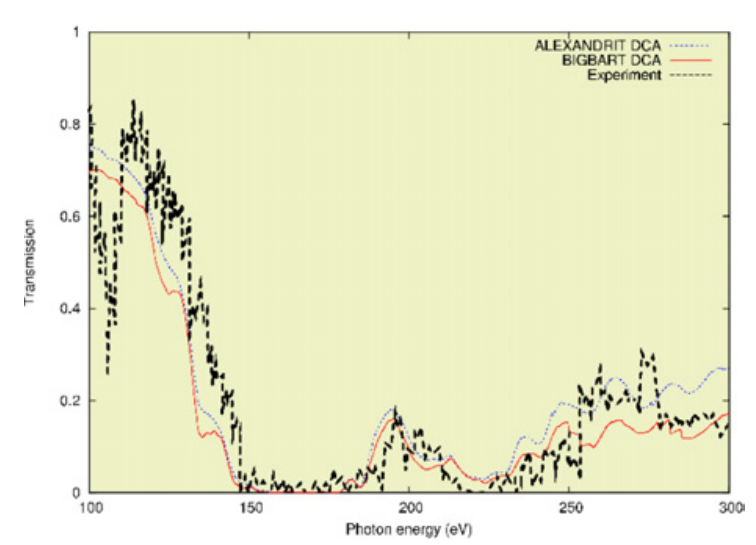

(a) Transmission using the DCA model.

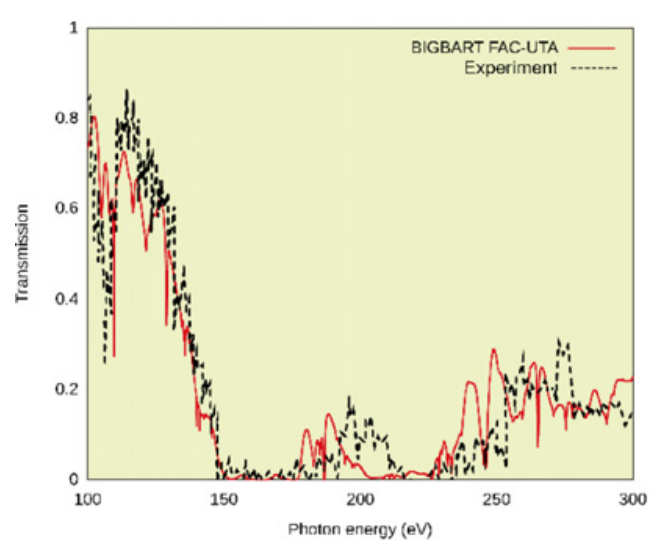

(b) Transmission using UTA model and taking atomic data from FAC.

Figure 3. Spectral transmision for a mixture of $\mathrm{Fe}$ and $\mathrm{NaF}$ at plasma conditions of $59 \mathrm{eV}$ and density of $11.3 \mathrm{mg} / \mathrm{cm}^{3}$. The equivalent areal density is $339 \mu \mathrm{g} / \mathrm{cm}^{2}$.

In figure 2 we compare the results from our EOS (full lines) using the SHM against the well-known Thomas-Fermi (TF) theory (dashed lines). Both models obtain the chemical potential of the system with a given temperature and density which is the key variable to get the other thermodynamic properties of the system, including the Helmholtz free energy. For figure 2 the pressure has been computed using two different sets of chemical potentials, one using the TF model and other using the SHM, showing great differences at low temperatures.

Finally it is possible to improve our analytical EOS using an empirical correction as described in [12] to make the thermodynamic data as close as possible to the avaliable experimental data and $a b$ initio MD simulations. 


\section{COMPARISON TO EXPERIMENTAL DATA}

In order to test mixtures calculations we have included in figure 3 the transmission coefficient for a mixture of iron with sodium fluoride compared with the experimental data described in [11]. Conditions determined were $59 \mathrm{eV}$ and $11.3 \mathrm{mg} / \mathrm{cm}^{3}$. We include non-relativistic (ALEXANDRIT DCA) and relativistic (bigbart DCA) hydrogenic calculations from previous versions used in the Instituto de Fusión Nuclear, figure 3(a).

The new FAC-UTA calculation in figure 3(b) shows in general a better fit, especially in the 100$150 \mathrm{eV}$ range, showing the absorption peak above $100 \mathrm{eV}$ which does not appear in the hydrogenic calculations.

\section{CONCLUSIONS AND FUTURE WORK}

The methodology and models used to compute EOS and opacities in the Instituto de Fusión Nuclear have been presented in this work. This data are stored in tables suited to be used in radiation hydrodynamic codes like ARWEN. These methods can obtain accurate atomic properties for several materials using well-known models and with a very fast algorithm.

Future work will include testing of RADIOM model in simulations and developing a full NLTE module in bigbart and also developing an EOS consistent with the atomic calculations for NLTE. Also a time-dependent ionization routine has been developed [15] to get the charge state evolution including NLTE effects and then corrections to the opacities and EOS must be applied to account for this NLTE effects.

We want to thank the financial support provided by the Spanish Ministerio de Educación y Cultura through the Plan Nacional no. ENE2009-09837 and also to the European Commission project SFINX-LASERLAB-II.

\section{References}

[1] C. García-Fernández, P. Velarde, M. Cotelo, IEEE Transactions on Plasma Science 38, 2359 (2010)

[2] M. F. Gu, Astrophys. J. 582, 1241 (2003)

[3] M. Busquet et al., High Energy Dens. Phys. 5, 270 (2009)

[4] G. Faussurier, C. Blancard, and P. Renaudin, High Energy Dens. Phys. 4, 114 (2008)

[5] A. G. de la Varga et al., High Energy Dens. Phys. 7, 163 (2011)

[6] R. D. Cowan, The theory of atomic structure and spectra, University of California Press (1981)

[7] Yu. Ralchenko, A.E. Kramida, J. Reader, and NIST ASD Team (2010)

[8] R. M. More, Appliead atomic collision physics, vol. 2, Academic Press, New York (1982)

[9] D. Salzmann, Atomic physics in hot plasmas, Oxford University Press, New York (1998)

[10] M. S. Dimitrijevic, N Konjevic, Astron. Astrophys. 172, 345 (1987)

[11] P. T. Springer et al., Phys. Rev. Letters 69, 3735 (1992)

[12] M. Cotelo, P. Velarde, A. G. de La Varga, C. García-Fernández, Astrophysics and Space Science (2010)

[13] V. Recoules and S. Mazevet, Phys. Rev. B 80, 064110 (2009)

[14] R.M. More, K.H. Warren, D.A. Young, and G.B. Zimmerman, Phys. Fluids 31, 3059 (1988)

[15] F. de Gaufridy, P. Velarde, D. Portillo, E. Oliva, C. Garcia, M. Cotelo, A. G. de la Varga, P. Zeitoun, S. Kazamias, K. Cassou and O. Guilbaud, High Energy Dens. Phys. 7, 294 (2011) 